\title{
Aktuelle Daten zu neuen Therapieoptionen
}

\section{Stammzelltransplantation \& alternative Behandlungsschemata beim Multiplen Myelom}

Eine wachsende Zahl an Behandlungsmöglichkeiten befindet sich beim Multiplen Myelom in klinischer Testung. Im Rahmen des Post-ASH 2011 Treffens Mitte Jänner in Salzburg rekapitulierte Univ.-Prof. Dr. Heinz Ludwig, Zentrum für Onkologie und Hämatologie, Wilhelminenspital Wien, die beim Jahreskongress der American Society of Hematology (ASH) 2010 in Orlando vorgestellten Studienergebnisse in diesem Forschungsbereich.

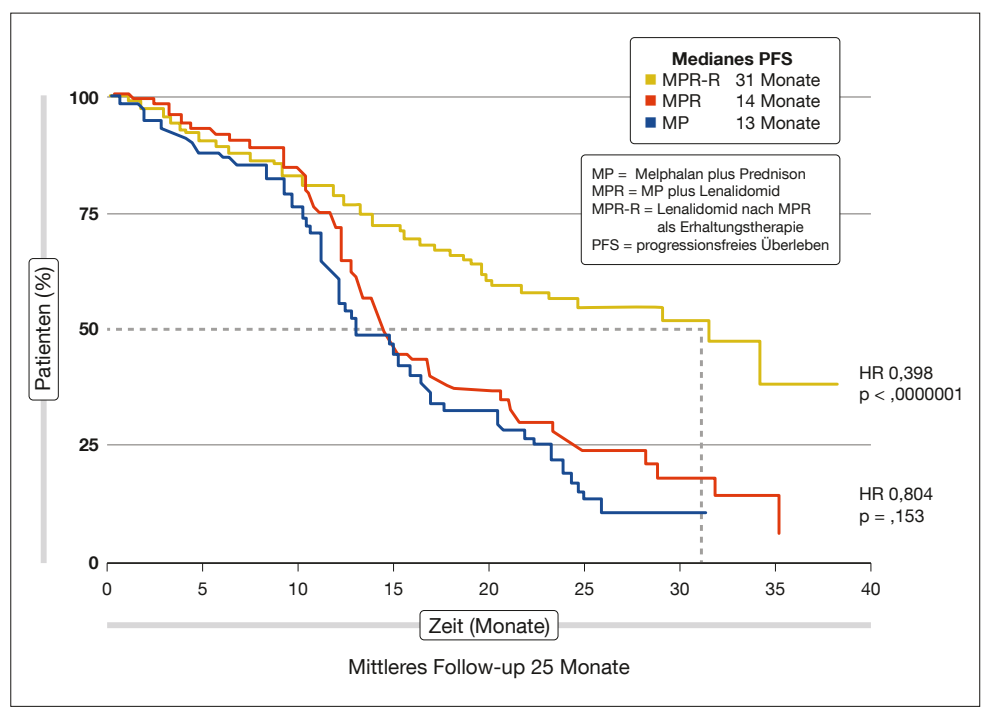

Abb. 1: MM-015-Studie: 60 prozentige Reduktion des Progressionsrisikos durch Lenalidomid in der Erhaltungstherapie (7).
Der Vergleich einer Tandem-ASCT (autologe Stammzelltransplantation) mit einem Schema aus ASCT gefolgt von nicht myeloablativer allogener Transplantation bei Patienten mit Standardrisiko stand im Mittelpunkt einer Studie von Krishnan et al. (1). „Die Gesamtansprechraten nach 56 Tagen unterschieden sich nicht signifikant", berichtete Ludwig ( $p=0,9)$. Dementsprechend resultierten identische Überlebensraten mit einem progressionsfreien Überleben (PFS) von 46 (TandemASCT) bzw. 43 Prozent und einem Gesamtüberleben (OS) von 80 bzw. 77 Prozent nach drei Jahren.

Eine Bortezomib-basierte Induktionsund Konsolidierungstherapie evaluierte die randomisierte Phase-III-Studie von Cavo (2). Dabei erhielten die Teilnehmer vor und nach einer Tandem-ASCT entweder nur Thalidomid plus Dexamethason (TD) oder zusätzlich Bortezomib (VTD). Der Anteil der Patienten, die mindestens eine fast vollständige Remission (nCR) erzielten, war in der VTD-Gruppe in allen Behandlungsphasen signifikant höher. Ein PFS-Vorteil ergab sich in allen Patientensubgruppen.

Terragna et al. analysierten die molekulare Remissionsinduktion durch die Konsolidierungstherapie in dieser Studie (3). Demnach konnte eine PCR-Negativität nach der Konsolidierung bei 67 (VTD) vs. 52 Prozent (TD) dokumentiert werden $(p=0,05)$. Ebenso nahm die Tumorlast im experimentellen Arm deutlich stärker $\mathrm{ab}(\mathrm{p}=0,05)$.

\section{Optionen für die Erhaltungstherapie}

Die endgültige Analyse der randomisierten, placebokontrollierten Phase-III-Studie IFM 2005-02 mit 614 Patienten, die eine Erhaltungstherapie mit Lenalidomid nach ASCT prüfte, wurde von Attal vorgestellt (4). Im aktiven Erhaltungstherapie-Arm lag das PFS ab der Randomisierung bei 42 Monaten, in der Placebogruppe dagegen nur bei 24 Monaten ( $p<0,00000001)$. Ludwig: „Der PFSBenefit wurde unabhängig von der Art der Induktionstherapie und dem Responsestatus beobachtet." Eine multivariate Analyse identifizierte die Therapie mit Lenalidomid sowie das Ansprechen auf die Konsolidierung als prognostisch relevant für das progressionsfreie Überleben. Das FünfjahresOS unterschied sich nicht zwischen den beiden Gruppen (beide $81 \%$ ).

Die HOVON-65/GMMG-HD4-Studie verglich eine Induktion mit Bortezomib, Doxorubicin und Dexamethason (PAD) mit dem Standard VAD (Vincristin, Adriamycin, Dexamethason) vor einer autologen Transplantation (5). „Im experimentellen Arm überstiegen die Ansprechraten nach Induktion und Hochdosis-Melphalan jene im
Standardarm signifikant", erläuterte Ludwig. Ein signifikanter Überlebensvorteil wurde im PAD-Arm sowohl hinsichtlich des PFS $(\mathrm{p}=0,005)$ als auch des OS $(p=0,02)$ verzeichnet. Die in der experimentellen Gruppe durchgeführte Erhaltungstherapie mit Bortezomib erwies sich im Vergleich zur Thalidomid-Maintenance im VAD-Kollektiv als gut verträglich.

Eine Bortezomib-Erhaltungstherapie nach drei Bortezomib-basierten Induktionsschemata (in Kombination mit Dexamethason, Dexamethason und Thalidomid oder Melphalan und Prednison) untersuchte eine Studie an neu diagnostizierten älteren Myelompatienten, bei denen keine Transplantation durchgeführt werden konnte (6). Nach Induktion und Erhaltung fanden sich in allen drei Armen vergleichbare Ansprechraten (Gesamtansprechen 71\%, 79\% und 73\%). Die Verträglichkeit der Bortezomib-Maintenance erwies sich als gut.

Die MM-015-Studie verglich Melphalan plus Prednison (MP) mit MP plus Lenalidomid (MPR), wobei Lenalidomid in einem dritten Arm nach MPR als Erhaltungstherapie eingesetzt wurde (MPRR) (7). Während sich das PFS unter MP und MPR nicht unterschied, bedingte die Erhaltungstherapie eine massive Verbesserung dieses Endpunkts. „MPR-R senkte das Progressionsrisiko um 60 Prozent", betonte Ludwig (medianes PFS 31 vs. 13 Monate; $\mathrm{p}<0,0000001 ; A b b .1)$.

Dr. Judith Moser

Quelle: Vortrag im Rahmen des Post-ASH 2011, 14. Jänner 2010, Salzburg

\section{LITERATUR}

1 Krishnan A et al., ASH 2010, Abstract 41 2 Cavo M et al., ASH 2010, Abstract 42 3 Terragna C et al., ASH 2010, Abstract 861 4 Attal M et al., ASH 2010, Abstract 310 5 Sonneveld P et al., ASH 2010, Abstract 40 6 Niesvizky R et al., ASH 2010, Abstract 619 7 Palumbo A et al., ASH 2010, Abstract 622 